\title{
Anabases
}

ANABASES Traditions et réceptions de l'Antiquité

13 | 2011

Varia

\section{Enjeux du traité hippocratique Des airs, des eaux et des lieux en 1800 : autour de l'édition de Coray}

Paul Demont

\section{(2) OpenEdition}

\section{Journals}

Édition électronique

URL : http://journals.openedition.org/anabases/1867

DOI : 10.4000/anabases. 1867

ISSN : 2256-9421

Éditeur

E.R.A.S.M.E.

\section{Édition imprimée}

Date de publication : 1 mars 2011

Pagination : 157-171

ISSN : 1774-4296

\section{Référence électronique}

Paul Demont, «Enjeux du traité hippocratique Des airs, des eaux et des lieux en 1800 : autour de

l'édition de Coray », Anabases [En ligne], 13 | 2011, mis en ligne le 01 mars 2014, consulté le 20 octobre 2019. URL : http://journals.openedition.org/anabases/1867 ; DOI : 10.4000/anabases. 1867

Ce document a été généré automatiquement le 20 octobre 2019

(c) Anabases 


\title{
Enjeux du traité hippocratique Des airs, des eaux et des lieux en 1800 : autour de l'édition de Coray
}

\author{
Paul Demont
}

1 Le traité hippocratique Airs, Eaux, Lieux fut rédigé probablement dans la seconde moitié $d u v^{e}$ siècle avant notre ère. L'une des éditions les plus importantes qui en aient été proposées est celle que le grec Adiamantos, ou Diamantos ou, dans la version francisée de son prénom, Diamant ou Adamance Coray, a publiée en 1800 à Paris, et rééditée en grec, pour les étudiants grecs de médecine, en 1816. Beaucoup de travaux de grande qualité ont déjà été consacrés en Grèce et en France à Coray et à cette édition : on en aura le meilleur aperçu dans l'édition du traité hippocratique procurée par Jacques Jouanna ${ }^{1}$ et dans les actes du dernier colloque qui, à ma connaissance, lui a été consacré, à Montpellier, en $1998^{2}$.

2 La Grèce était alors sous occupation turque. Coray, né en 1748 à Smyrne dans une famille de négociants de Chio, est arrivé en France en 1782 à 34 ans pour fuir le despotisme, mais aussi probablement le négoce (et peut-être aussi le mariage). Il étudia d'abord la médecine à l'université de Montpellier, et notamment la médecine hippocratique, fondée sur la nature, que la Faculté de Montpellier défendait avec les Encyclopédistes, puis vécut à Paris, où, pendant la tourmente révolutionnaire, il prépara son édition, au milieu d'autres travaux, souvent alimentaires, qui montrent et sa puissance de travail, et que la médecine n'était pas son intérêt unique : une édition des Caractères de Théophraste l'année précédente, ensuite, sur commande de Bonaparte, et avec d'autres, une édition de la Géographie de Strabon, tout en se lançant dans sa grande entreprise de la Bibliothèque hellénique.

Son édition d'Airs, Eaux, Lieux ${ }^{3}$, à laquelle il semble avoir travaillé dès 1788 , sera ici envisagée sous cinq aspects différents : c'est un travail de philologie (ou, pour employer le vocabulaire de Coray, de " critique ») ; c'est l'œuvre d'un Grec ; mais est-ce celle d'un Européen? C'est cependant aussi un travail médical. Et enfin, quels sens peut prendre la théorie des climats autour de 1800 ? 
Le rôle de Coray comme philologue et éditeur est bien connu. Émile Littré, l'éditeur de l'ensemble de ce qu'on appelle la Collection hippocratique, écrit quarante ans plus tard dans son introduction à notre traité: "En sa triple qualité de Grec, de médecin et d'helléniste, Coray était plus propre qu'aucun autre à donner une édition du Traité des Airs, des Eaux et des Lieux; et j'ai cru ne pouvoir trop puiser à une source d'érudition aussi abondante et aussi sûre ${ }^{4}$." Les éditeurs ultérieurs ont reconnu aussi leur dette. Jacques Jouanna, qui résume les différents apports de Coray, notamment son utilisation de notes d'un érudit italien qui possédait un manuscrit ancien, sa connaissance de la tradition indirecte et son attention à l'établissement du texte, écrit : « Nul philologue, par les seules ressources de son intelligence et de son acharnement, n'a fait progresser autant que lui la compréhension du texte ${ }^{5}$.» Jacques Jouanna a consacré de longues semaines de travail à explorer la bibliothèque de Coray à Chio, ce qui lui a permis de découvrir à quel point Coray, bien après cette édition, a continué de s'intéresser à Hippocrate et il a écrit deux beaux articles sur cet intérêt de "philologue » et sur la façon dont Coray a édité le traitét, ce qui nous permettra d'être bref sur cet aspect essentiel.

5 Il suffit d'ailleurs de prendre une page de l'édition Jouanna pour voir que le meilleur éditeur actuel de la Collection hippocratique dialogue très souvent avec Coray. Dans la description de la Scythie et de ses habitants, sont mentionnés les Monts Rhipées, au nord du pays, dans une description dont voici la traduction par J. Jouanna, avec une partie de son apparat critique $^{7}$ :

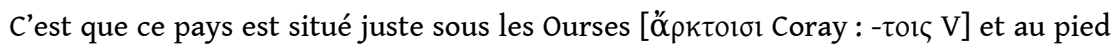

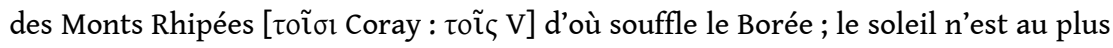
près qu'à la fin de sa course, quand il arrive à son circuit estival (= solstice d'été), et

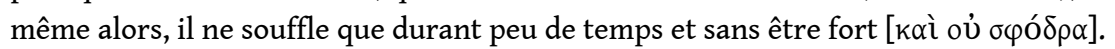

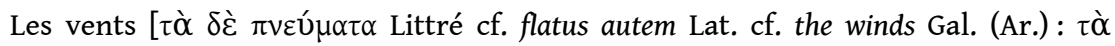
$\delta \varepsilon เ \pi v \varepsilon \dot{u} \mu \alpha \tau \alpha \mathrm{V}$ ] soufflant des régions chaudes ne parviennent pas [oủk Littré cf. non Lat. cf. do not Gal. (Ar.) : om. V], sinon rarement et faiblement ; en revanche, à partir des Ourses (= du nord), soufflent continuellement des vents froids, dus à la neige, à la glace et aux pluies abondantes; ces conditions climatiques ne quittent jamais les monts (Rhipées) ; aussi sont-ils inhabitables [ảoıkntó prop. Coray. (I, 160 et II,

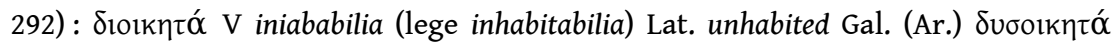
edd. ab Ald.].

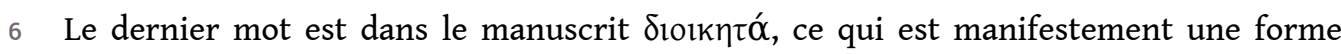
impossible. Coray, au moyen à la fois d'une très bonne connaissance de la littérature grecque (à une époque où on ne disposait pas du TLG!) ${ }^{8}$ et d'une méthode d'analyse des fautes de copie toujours valable (mélectures d'onciales 糔/A), a proposé une correction qui est quasiment certaine, reprise par Jacques Jouanna, que confirment le commentaire de Galien et la traduction latine ancienne (deux documents dont Coray ne

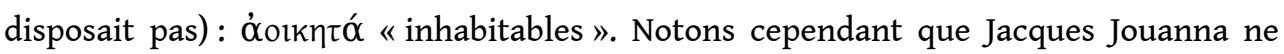
suit pas toujours Coray. Et s'il estime souvent devoir discuter en détail son désaccord", ici il ne dit rien du passage qui précède immédiatement la correction que nous venons de citer, et pour lequel Coray avait proposé dans son commentaire, un autre texte, avec l'explication suivante, très différente de celle de Jacques Jouanna ${ }^{10}$ :

Je ne crois pas avoir manqué au devoir d'un éditeur en changeant le mot

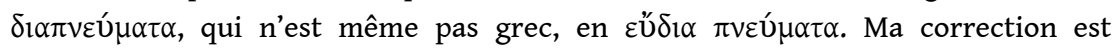

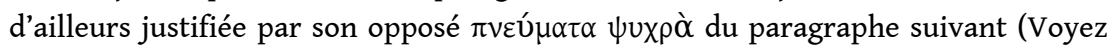
aussi not. LXI, 1. 2, p. 157). 
7 Jacques Jouanna édite ici un texte très différent, qui reprend deux corrections de Littré introduisant une proposition supplémentaire, ce qui oblige à introduire aussi une négation supplémentaire dans cette nouvelle proposition. Ce texte est appuyé par les documents que Coray ne connaissait pas, le commentaire de Galien et la traduction latine ancienne. La solution de Coray, plus économique, qui conserve le lien entre oủ $\sigma \varphi o ́ \delta \rho \alpha$ et la suite, mérite cependant peut-être de ne pas tomber dans l'oubli.

Coray, on le verra, n'intègre pas toujours dans son texte, comme il le fait ici, les corrections qu'il propose en note. À cet égard, pour reprendre une observation de Jacques Jouanna, Coray se situe à la frontière entre deux époques de la philologie : il n'ose pas encore établir le texte sur nouveaux frais, et propose seulement l'édition reçue de Foes, avec des améliorations. Il est aussi à la frontière d'une façon plus importante : Hippocrate, avec lui, devient un auteur pour philologues et historiens, et plus guère pour médecins. Plus tard, l'édition d'Émile Littré connaîtra une évolution comparable.

9 Notons enfin dans l'apparat critique de cette même page deux corrections de Coray, retenues par les éditeurs suivants, qui visent à rétablir des ionismes. Coray était très attentif au dialecte, un des points sur lequel il dit dès la première édition avoir amélioré son texte de base, qui est l'édition de Foes ${ }^{11}$. Il signale aussi plus tard que l'édition de 1816, du point de vue du texte, est encore modifiée en ce sens. Il est attentif en général à la langue, et il estimait avoir sur ce point une compétence supérieure aux non-Grecs. Lui était, comme le note finement Littré, helléniste et Grec! Ici nous dépassons la simple philologie et abordons le second aspect de l'édition de Coray.

Coray l'émigré, en travaillant à son édition, vit en quelque sorte un peu en Grèce, tout en éprouvant douloureusement l'éloignement de son pays natal. Tout se passe comme si travailler sur Airs, Eaux, Lieux était pour lui vital ${ }^{12}$ :

En quittant mon malheureux pays, je n'avais encore aucune idée de la médecine, qui pût m'inspirer le désir de vérifier ces observations sur les lieux mêmes. Les regrets que j'en ai à présent sont d'autant plus douloureux que peut-être je n'aurai jamais la satisfaction de revoir ce pays chéri, jadis berceau des sciences et des arts, et qui est aujourd'hui infecté de la barbarie d'une nation féroce. Mais, je le répète, celui qui nous donnerait une topographie médicale bien détaillée de tous les cantons de la Grèce ferait le meilleur commentaire des ouvrages d'Hippocrate.

D'emblée il avait présenté Hippocrate au travail « dans un coin de la Grèce ${ }^{13}$ ». La Grèce dans sa réalité physique renaît et renaîtrait en lisant Hippocrate, tout comme Hippocrate ne redeviendrait vraiment vivant et pertinent qu'en parcourant la Grèce, même si l'on peut et si l'on doit étendre ses remarques sur la Grèce à « la topographie des différents pays de l'Europe ${ }^{14}$ ", notons bien, « de l'Europe ». C'est peut-être aussi en ce sens qu'il faut lire le développement sur les vents de la Grèce qui occupent les p. LXXIII-LXXXIII du Discours préliminaire ${ }^{15}$.

12 Il y a donc un privilège grec pour comprendre Hippocrate, et l'Europe a ainsi quelque chose à apprendre de la Grèce. Coray réclame alors par voie de conséquence la connaissance de la Grèce contemporaine, de sa langue et stigmatise au passage la prononciation érasmienne (ibid.) :

Pour tirer tout le parti d'une pareille topographie, il faudrait que celui qui aurait le courage de l'entreprendre y apportât, outre les connaissances physiques et médicales, la connaissance profonde de la langue grecque d'Hippocrate et de plus de celle que parlent les Grecs modernes. Il faudrait surtout, en abandonnant les préjugés européens, qu'il se résolût à prononcer l'une et l'autre comme on les 
prononce aujourd'hui en Grèce. Prétendre que les Européens prononcent mieux le grec que les natifs de la Grèce est une extravagance digne du $\mathrm{xvI}^{\mathrm{e}}$ siècle, qui la vit naître, mais qui aurait déjà dû disparaitre devant les lumières du nôtre. pour Erasme une "mauvaise plaisanterie", est que les voyageurs européens ne comprennent rien et qu'ils ne sont pas compris. Ainsi, par la continuité de la langue, par la résurrection d'Hippocrate et par la continuité du paysage et du climat, la Grèce moderne (qu'il représente à Paris, bien qu'elle n'existe pas) rejoint la Grèce ancienne, le berceau des sciences et des arts que représente Hippocrate.

Cest dans cette perspective que Coray s'adresse à ses compatriotes, « qui lui ont fourn les moyens de publier cet ouvrage" (il s'agit de négociants grecs qui furent ses mécènes), à la fin de son introduction, et se livre à un résumé remarquable de l'histoire de la Grèce ${ }^{16}$ :

Je vous félicite de ce que votre zèle dément, de la manière la plus victorieuse, toutes les absurdes calomnies par lesquelles, dans ces derniers temps, des personnes qui par leurs lumières devraient s'intéresser le plus à nos malheurs, ont cherché à dénigrer notre nation. On aura beau dire: vous n'avez point dégénéré de vos illustres ancêtres, le sang grec coule encore dans vos veines; il n'attend qu'un heureux concours de circonstances pour prouver à l'univers que vos chaînes n'ont pas été votre ouvrage, et que, loin de les avoir portées avec une stupide résignation, vous avez été la seule nation subjuguée qui ait voué une haine éternelle à ses tyrans, haine transmise de père en fils, nourrie et conservée, dans vos cœurs indignés, comme un héritage sacré. Des despotes transplantés de l'ancienne Rome, après avoir, par une administration aussi stupide que tyrannique, relâché tous les ressorts de la société, entravé l'influence du plus beau des climats, souillé, ébranlé leur trône par les crimes les plus affreux, ont fini par vous livrer à des tyrans encore plus stupides et plus féroces.

Il s'en prend ici, entre autres, à un Dr Weikard, estimable membre des Lumières, mais qui avait développé une théorie très curieuse sur le lien entre la stupidité des Grecs modernes et la disparition de la consommation du vin en Grèce à la suite de l'arrachage des vignes par les Ottomans. D'où la pointe pleine d'humour de la citation finale, donnée uniquement en grec, et sans référence, mais dont voici une traduction : « Même si tu me manges jusqu'à la racine, je porterai tout de même encore du fruit en quantité suffisante pour en faire une libation quand on te sacrifiera, toi, le bouc ${ }^{17}$." Mais surtout, il applique manifestement à l'histoire grecque la tension qui caractérise la fin du traité hippocratique, et sur laquelle je reviendrai, entre l'influence du climat et celle des institutions politiques.

De nouvelles institutions politiques correspondant mieux au climat de la Grèce pourront donc lui rendre sa splendeur perdue. C'est d'ailleurs déjà en bonne voie, selon ce qu'il déclare trois ans plus tard dans son célèbre Mémoire sur l'état actuel de la civilisation dans la Grèce, qu'il lit en 1803 à la Société des observateurs de l'homme ${ }^{18}$.

On voit ici que Coray a au moins deux publics, «les Européens» et les «natifs de la Grèce ». L'Europe, la Grèce. Au moins deux publics, parce que chacun d'entre eux se divise à l'infini, bien sûr, non sans entraîner quelques contradictions parfois. Mais pour en rester à ces deux publics, il vaut la peine de tenter de préciser un peu le rapport qu'ils ont entre eux. On connaît les célèbres passages du traité hippocratique dans lesquels le médecin ajoute, à l'influence du climat, celle des lois et des mœurs. Il y en a deux, en particulier, très proches l'un de l'autre, dans les chapitres XVI et XXIII-XXIV actuels, que voici dans la traduction de Coray. Ils offrent une variation peu soulignée, 
mais intéressante dans notre perspective d'aujourd'hui, surtout dans la traduction qu'en donne Coray ${ }^{19}$ :

LXXXVIII $^{20}$. Une grande preuve de ce que j'avance, c'est qu'en Asie même tous ceux des Grecs et des Barbares qui se gouvernent par leurs propres lois, sans être soumis à des despotes, et qui par conséquent travaillent pour eux-mêmes, sont les hommes les plus belliqueux de tous. C'est qu'ils ne s'exposent que pour eux-mêmes, et que ce sont eux qui reçoivent le prix de leur courage, ou qui portent la peine de leur lâcheté.

$[\ldots]$

$\mathrm{CXVII}^{21}$. C'est sans doute la nature du climat qui rend les Européens plus belliqueux que les Asiatiques, mais la forme du gouvernement y contribue aussi. Les premiers ne sont pas gouvernés par des rois comme le sont les autres, et j'ai déjà observé que partout où l'on est soumis à des rois, on est nécessairement très lâche; parce que, quand on a l'âme asservie, on ne se soucie point d'exposer sans nécessité sa vie pour augmenter la puissance d'un autre. CXVIII. Les Européens, au contraire, gouvernés par leurs propres lois ${ }^{22}$, affrontent d'autant plus volontiers les dangers qu'ils ne s'y exposent que pour eux-mêmes, et que ce sont eux seuls qui recueillent l'honneur et le fruit de leurs victoires. Tant il est vrai que les lois influent singulièrement sur le courage. En comparant les Européens avec les Asiatiques, je n'ai parlé que d'une manière générale.

Voici l'excellent commentaire du premier passage par Coray, avec un ajout significatif ${ }^{23}$ :

LXXXVIII, 1. Tous ceux des Grecs et des barbares qui se gouvernent par leurs propres lois, etc. Hippocrate pensait donc que l'influence du climat, quoique réelle, pouvait cependant être modifiée par la forme du gouvernement ou par toute autre cause morale. Il cite pour exemple les Grecs d'Asie, qui, malgré la nature du climat, étaient plus vaillants que leurs voisins, les Perses. Ils auraient fini par triompher de ces derniers, et affirmé leur liberté, s'ils eussent suivi le conseil de Thalès.

Coray justifie donc ici Hippocrate au moyen d'Hérodote ; le conseil de Thalès, on le sait, consistait à quitter le continent pour s'expatrier en Sardaigne, y fonder une colonie panionienne, afin de mieux résister au despotisme (I, 170). Inutile d'insister sur le parallélisme sous-jacent avec la situation de Coray lui-même, nouveau Thalès à Paris.

Le second passage concerne « les Européens » et « les Asiatiques » dans la traduction de Coray, qui est, notons-le, celle du texte avant correction. Certains exemples pris par Coray pour illustrer « les Européens » et « les Asiatiques » sont remarquables.

Du côté de l'Europe, il est impossible de ne pas le mentionner ici et aujourd'hui ${ }^{24}$, il y a la Suisse ${ }^{25}$ :

CXVII.5. [...] Le plus grand éloge qu'on puisse faire du gouvernement suisse, c'est cette maladie connue sous le nom de nostalgie ou de mal du pays, qui attaque de préférence les naturels de ces heureuses contrées, lorsqu'ils se trouvent en pays étranger. La liberté dont ils jouissent, jointe à la simplicité de leurs mœurs, fait naître en eux cette inquiétude et ce désir si vif de retourner dans leur patrie.

Du côté de l'Asie, il y a par exemple les Turcs, qui, soumis au despotisme, se battent sans vigueur. L'histoire toute récente a permis à Coray de vérifier l'analyse d'Hippocrate : les Russes ont battu les Turcs au terme d'une longue guerre (1787-1792) et imposé au Sultan un accord qui favorisa le développement de la diaspora et du commerce grecs. Les Russes, pourtant eux aussi soumis à un despotisme, mais moindre, sont plus favorisés par le climat que les Turcs, du point de vue du courage.

La Suisse, une Suisse assez rousseauiste, incarne donc une sorte de quintessence de l'Europe hippocratique pour Coray, on pourrait presque dire un double de la Grèce 
idéale dont le climat et les mœurs concordent dans le bon sens. Et les Suisses ont un sens de la nostalgie bien proche de celui qu'éprouve Coray lui-même.

Mais pourquoi la Suisse, et non la France? C'est que d'autres nations européennes, elles, plus puissantes, et peut-être gouvernées par des despotes elles aussi (cela, je l'ajoute, car Coray se garde d'être trop précis sur ce point), ont été corrompues par leurs mœurs. Il faut ici revenir au Discours préliminaire. Coray donne des exemples des transformations que la morale et les lois installent dans une population, notamment en Europe, en étendant une observation de Strabon à la situation à l'époque moderne, dans une gradation construite d'une façon caractéristique ${ }^{26}$ :

Strabon observe que les Scythes Nomades, tant vantés par Homère, à cause de leur amour pour la justice, étaient de son temps tellement pervertis, pour avoir embrassé le commerce maritime, qu'ils pillaient, assassinaient les étrangers, quoique d'ailleurs ils parussent plus policés. Quelqu'un s'imaginera peut-être que le commerce n'a pas, à beaucoup près, produit les mêmes effets chez les peuples modernes : mais qu'il songe à l'effusion de sang qui a accompagné la découverte de l'Amérique, et au trafic infâme des nègres que la culture de cette partie du monde a nécessité ; qu'ils songent à l'oppression que plusieurs nations européennes exercent encore aujourd'hui sur les malheureux indigènes de leurs possessions en Asie et en Afrique ; qu'ils songent enfin que ce n'est qu'aux avantages du commerce du Levant que les nations policées de l'Europe sacrifient sans pitié comme sans remords la liberté de la Grèce; et qu'ils osent après cela décider si l'on est aujourd'hui plus humain que ne l'étaient autrefois les Scythes.

La découverte de l'Amérique et le massacre des populations indigènes, puis la traite négrière, enfin l'indifférence devant l'asservissement de la Grèce : quelle gradation! Il semble, à lire ces protestations très émouvantes contre le mythe du progrès de la nature humaine, que Coray se fasse un malin plaisir de montrer que si, d'un côté, les Grecs ont été, en apparence, transformés par des siècles de despotisme, puis par la tyrannie turque, les Européens de leur côté ont aussi été corrompus par leurs mœurs commerciales (il corrigera cette attaque dans son Mémoire trois ans plus tard, peut-être pour ne pas trop heurter ses mécènes, par un éloge des effets positifs du commerce en Grèce). Ils sont, dans cette mesure, responsables eux aussi de l'état actuel de la Grèce. La conséquence implicite en est que les Grecs peuvent et doivent retrouver leur ancien état, et que l'Europe peut et doit retrouver aussi, pour lutter avec les natifs de la Grèce, la vertu et le courage qui la caractérisent fondamentalement.

Autre difficulté : pour Hippocrate, il y a en Asie une région médiane qui a le climat le plus beau (XII, 2-3, p. 219-220 Jouanna), et, comme la comparaison avec un passage très proche d'Hérodote (I, 142, 1-2) le montre d'une façon certaine, il s'agit notamment, sinon exclusivement, de l'Ionie du Panionion. Or certains peuples en Asie ont néanmoins un caractère qu'on pourrait qualifier d'européen, en raison de leur mode de gouvernement (XVI, 5, p. 229 Jouanna).

On a noté avec raison ${ }^{27}$, qu'Hippocrate parle alors de «Grecs ou Barbares », ce qui tend à effacer la distinction politique entre Grecs et Barbares au profit d'une distinction "scientifique " entre Européens et Asiatiques. Mais on voit aussi à quel point il est nécessaire, pour le médecin antique, de compléter l'influence du climat par l'influence des lois, afin de faire coïncider, notamment dans le cas de l'Asie mineure, les idéaux climatiques naturels avec les idéaux politiques et moraux. Or c'est aussi vrai pour le médecin moderne, qui voit justement dans l'Ionie, et dans l'île de sa famille, Chio, le point de départ de la régénération grecque. C'est ce qu'il déclare du moins dans son 
Mémoire ultérieur, et cela colore d'un contexte très contemporain les ionismes réintroduits par Coray dans le texte d'Hippocrate ${ }^{28}$ :

Une des choses les plus remarquables, et qui confirme en quelque manière la doctrine d'Hippocrate, sur l'influence des climats, c'est que les lumières dans la Grèce ancienne avaient commencé par l'Ionie, et que leur renaissance dans la Grèce moderne semblerait affecter la même marche.

[...]

Le dialecte ionique fut cultivé et perfectionné longtemps avant celui d'Athènes... Jusqu'au siècle d'Hérodote et d'Hippocrate, qui touche au siècle de Platon, il faut que le dialecte des Ioniens ait été regardé comme le plus parfait, puisque le père de l'histoire, comme celui de la médecine, le choisirent, pour y consacrer leurs immortels ouvrages, quoiqu'ils fussent l'un et l'autre doriens d'origine [...] J'ai dit que la renaissance des lettres dans la Grèce moderne semblerait affecter la même marche qu'avait prise leur naissance, en commençant par l'Ionie, et en se propageant successivement dans le reste de la Grèce. Car, quoique la révolution qui fait l'objet de mes observations soit commune à tous les Grecs, c'est dans la patrie même d'Homère, dans l'île de Chio, que la Grèce moderne a la satisfaction de voir, depuis quelques années, le premier établissement d'une espèce d'université ou d'école polytechnique. pour ses fautes de grec. Il relève notamment une confusion, à propos d'un malade des Epidémies, entre le nom propre Cyniscos, que lui, Coray, restitue (avec raison) et l'appellation de «Cynique » que croit lire Haller. Et il conclut : « Il faut être circonspect dans les jugements qu'on porte sur les ouvrages des Anciens, surtout quand on ne possède pas bien la langue dans laquelle ils ont écrit et qu'on n'a pas sans cesse sous les yeux l'ensemble de leur doctrine » (p. LVI). De la même façon, en raison d'une faute dans une traduction latine qu'il utilise, Haller s'est trompé en refusant à Hippocrate la paternité d'Airs, Eaux, Lieux. Vous vous référez à Hippocrate ? Eh bien mieux vaut savoir le lire, semble dire Coray. Sa connaissance du grec semble d'ailleurs avoir été un élément important de son succès lors de sa thèse, à en juger par le récit qu'il en fait au Protopsalte de Smyrne : « En réfutant les objections, j’ai exposé que beaucoup de choses que l'on croyait être des découvertes modernes, Hippocrate les avait sues deux mille ans auparavant. »

Cette phrase livre un aspect essentiel de Coray médecin : il tente une sorte de retour à Hippocrate, à l'Hippocrate authentique, à partir de la modernité. C'est d'ailleurs un aspect non négligeable de l'enseignement qu'il a suivi à la Faculté de Montpellier, et plus généralement de la présentation de la médecine dans l'Encyclopédie, présentation à laquelle cette Faculté a beaucoup contribué, que ce néo-hippocratisme, qui tente de dépasser les conflits entre partisans de la mécanique, de la chimie ou du vitalisme en croyant revenir à l'observation, à la raison et au respect de la nature, ces « forces de la nature » exaltées par les physiologues, et sur lesquelles, selon Sprengel, Hippocrate 
compte un peu trop. Citons notamment les noms de P.-J. Barthez et du maître de Coray, J.-Ch. Grimaud.

31 On peut prendre un exemple précis, à partir du Discours préliminaire, d'une tentative, plutôt peu réussie, d'utilisation médicale moderne d'Hippocrate par Coray. Hippocrate, remarque-t-il, après avoir considéré l'exposition des villes à un très grand nombre de vents, ne parle que de "deux états de l'atmosphère ", "savoir, de l'état austral et de l'état boréal ${ }^{31}$ ». Il y a là une difficulté réelle, qui est présentée ainsi par Jacques Jouanna: «Bien que l'auteur établisse quatre orientations possibles de la cité, entraînant quatre états sanitaires possibles dans la population d'une cité, les constitutions des habitants ne répondent en réalité qu'à deux types possibles, ainsi que les maladies qui les affectent. Ce sont les deux types d'hommes opposés correspondant aux deux orientations opposées nord/sud ${ }^{32}$. Cette difficulté est explicable, montre-t-il, si l'on considère que l'auteur du traité n'a pas à sa disposition un système de quatre humeurs comparable à celui du traité Nature de l'homme, mais un système à deux humeurs, bile et phlegme, associées traditionnellement, et respectivement, à l'été et à l'hiver. Si bien que les orientations est/ouest « ne donnent lieu qu'à des remarques sur l'aspect extérieur des habitants, et non sur leur constitution interne».

Coray a une autre explication. L'état austral et l'état boréal correspondent à une division binaire. "Or, il est prouvé par les observations de Sydenham qu'en Europe, comme en Grèce, les maladies épidémiques, outre l'influence qu'elles reçoivent d'une ou de plusieurs saisons, ont encore un caractère semestral, si je puis m'exprimer ainsi, caractère que leur impriment l'équinoxe du printemps et celui de l'automne. [...] $\mathrm{Ce}$ qu'il y a de certain, c'est que cet effet existe ${ }^{33}$. " L'Hippocrate anglais, Sydenham ${ }^{34}$, et ses « observations » à la manière des Épidémies, sont invoqués à l'appui des analyses du traité du $v^{e}$ siècle et pour mieux les comprendre.

Venons-en enfin à ce qui a surtout fait la fortune du traité hippocratique, la théorie du climat, et à ses usages au tournant des XVIII ${ }^{\mathrm{e}}$ et XIX⿸e siècles.

Tout lecteur français pense bien sûr à Montesquieu. De fait, la tonalité hippocratique d'une réflexion comme celle que Montesquieu propose sur les Anglais dans l'une de ses Pensées semble évidente, avec le double jeu du climat et des lois ${ }^{35}$ :

Le caractère des Anglais marqué dans tous les temps est une certaine impatience que le climat leur donne, et qui ne leur permet ni d'agir longtemps de la même manière, ni de souffrir longtemps les mêmes choses: caractère qui n'est point grand en lui-même, mais qui peut le devenir beaucoup, lorsqu'il n'est point mêlé avec de la faiblesse, mais avec ce courage que donnent le climat, la liberté et les lois.

Coray lui aussi y pense, mais pour le critiquer. Il reproche en effet à Montesquieu de ne pas avoir avoué sa dette envers le Père de la Médecine et envers ce texte génial qui est utile à la fois au médecin, à l'historien, au cosmographe et au politique, car ils y trouvent chacun « les premiers fondements » de leurs sciences respectives ${ }^{36}$ :

Ce jugement a été justifié de nos jours par un autre ouvrage de génie (L'Esprit des lois), dont l'auteur n'aurait rien retranché de sa propre gloire, s'il avait eu le noble courage de faire honneur au médecin grec du principe fécond qui lui fournit l'idée de son travail, et ce qui en fut la base.

Cependant, cette critique ne semble guère justifiée. Si Montesquieu ne mentionne pas Hippocrate, c'est surtout, selon toute vraisemblance, parce qu'il ne se fonde pas sur lui. Il suffit de lire les premières lignes du chapitre II du livre XIV de L'Esprit des lois («Combien les hommes sont différents sous les divers climats») pour voir toute la 
différence entre son analyse cardiologique et fibrillaire des effets du climat (ou plutôt, d'ailleurs, de l'air) et la théorie hippocratique, qui n'offre absolument aucun point de comparaison précis :

L'air froid resserre les extrémités des fibres extérieures de notre corps, cela augmente leur ressort, et favorise le retour du sang des extrémités vers le cœur : il diminue la longueur de ces mêmes fibres, il augmente donc encore par là leur force. L'air chaud au contraire relâche les extrémités des fibres et les allonge. Il diminue donc leur force et leur ressort. On a donc plus de vigueur dans les climats froids. L'action du cœur et la réaction des extrémités des fibres s'y font mieux, les liqueurs sont mieux en équilibre, le sang est plus déterminé vers le cœur, et réciproquement le cœur a plus de puissance. Cette force plus grande doit produire bien des effets; par exemple, plus de confiance en soi-même, c'est-à-dire plus de courage.

Montesquieu est ici beaucoup plus proche d'un médecin anglais ami de Swift, John Arbuthnot, que d'Hippocrate. Arbuthnot publie en 1733 un essai sur Les effets de l'air sur les corps humains, ouvrage traduit en 1742 à Montpellier, justement, et dédié alors, notons-le, à Helvétius. Je cite une phrase de son introduction dans laquelle il invoque, lui, le patronage d'Hippocrate : «Le docteur Sydenham, rempli du génie d'Hippocrate, nous a laissé des Epidémies écrites sur le modèle de ce Prince de la Médecine, contenant une histoire des maladies aiguës comme dépendantes de la constitution de la saison. » Montesquieu aurait pu se livrer aussi à cet hommage très convenu. Mais Arbuthnot étudie ensuite minutieusement les ingrédients, les propriétés, les qualités, la nature, les usages, l'influence de l'air, les fièvres pestilentielles transmises par l'air et les explosions naturelles de l'air, et Hippocrate n'est invoqué que dans l'introduction; le reste du livre explore la physiologie nouvelle des fibres, et tente de mesurer les composants de l'air. De cela, Coray ne parle pas. Voici quelques lignes d'Arbuthnot qu'on pourra aisément comparer au texte de Montesquieu :

XV. Le froid condense l'air proportionnellement à ses degrés. II contracte les fibres animales et les fluides aussi loin qu'il les pénètre : les dimensions des animaux, réellement moindres dans le froid, démontrent ce fait. Le froid resserre les fibres non seulement par sa qualité condensante, mais encore en congelant l'humidité de l'air. L'extrême froid agit sur le corps, en manière d'aiguillon, produisant d'abord un picotement, et ensuite une chaleur brûlante, ou un léger degré d'inflammation, dans les parties qui y font exposées. Il produit par son irritation, par le resserrement des fibres et la condensation des fluides, la force, et l'activité, très sensibles à certaines personnes dans un temps clair de gelée. Si les effets de l'air froid font si considérables sur la surface du corps humain, combien plus le sont-ils sur le poumon, où le sang est beaucoup plus chaud.

Cela dit, Montesquieu et Coray sont pris dans la même difficulté, qui était déjà celle d'Hippocrate. Y a-t-il un déterminisme du climat ? Coray évoque les critiques virulentes émises par David Hume et, en France, par Helvétius (mais aussi par l'Église, dont Coray ne parle pas, mais à laquelle Montesquieu cherche à répondre dans la deuxième édition de L'Esprit des lois), contre cette idée, qui pourrait enlever toute importance à l'éducation ${ }^{37}$.

Les Chinois, ajoute encore Hume, ont par tout leur vaste empire le même caractère national, quoique leur climat ne soit point partout le même. Fallait-il aller chercher des exemples chez une nation si éloignée de nous, pour établir une vérité qui, sans détruire l'influence du climat, prouve tout au plus que cette influence peut être modifiée par des causes morales? Quoique je ne connaisse les Chinois que par les relations des voyageurs, qui les connaissent eux-mêmes fort peu, j'ose affirmer qu'il en est de la Chine comme de tous les autres pays que nous connaissons de manière plus particulière. Le caractère national de ce peuple, le même quant aux causes 
morales qui l'ont formé, doit présenter par la force des causes physiques autant de nuances qu'il y a de provinces différentes; de même qu'en France, pays beaucoup moins étendu que la chine, et qui présente aussi un caractère national bien prononcé, on observe une différence sensible entre un Languedocien et un Normand, un Provençal et un Breton. Et il n'est pas non plus vrai, comme le prétend Hume, que les Juifs soient partout les mêmes, quoique dispersés sur tout le globe. À travers cette uniformité frappante, qui est un effet des causes morales [...], on distingue bien, pour peu qu'on y fasse attention, un Juif allemand d'un Juif portugais. Je ne m'arrêterai pas plus longtemps aux objections de cet illustre philosophe [Note: Par la même raison, je crois pouvoir me dispenser de parler du système d'Helvétius, qui rapporte tout à l'éducation].

On notera au passage que pour Coray les différences régionales ne peuvent venir que $\mathrm{du}$ climat. Mais on notera aussi que, comme Montesquieu, il refuse l'idée d'une opposition absolue entre les causes naturelles et les causes morales. Coray répond ainsi au reproche que tout lecteur ne peut manquer de faire à Hippocrate lui-même ${ }^{38}$ :

Et que l'on ne m'accuse point de faire un cercle vicieux, si après avoir fait dépendre le moral de l'homme des causes physiques, je considère ici ces dernières comme dépendantes en quelque manière du moral. Ce cercle est dans la nature-même de l'homme, il est la suite nécessaire de cette intime liaison entre l'esprit et le corps.

Le caractère de déterminisme que peut avoir le climat se manifeste avec une particulière acuité, chez Montesquieu, dans la question de l'esclavage. Montesquieu, tout en affirmant que les lois doivent corriger les effets néfastes du climat, et tout en condamnant très ironiquement et très vigoureusement les mauvaises justifications qu'on apportait de cette pratique dans le très célèbre chapitre $\mathrm{V}$ du livre $\mathrm{XV}$, étudie ensuite, ce qui est beaucoup moins connu, la "véritable origine ", climatique, du droit de l'esclavage, qui entraîne son «inutilité » parmi nous : "Il faut donc borner la servitude naturelle à de certains pays particuliers de la terre. Dans tous les autres, il me semble que, quelques pénibles que soient les travaux que la société y exige, on peut tout faire avec des hommes libres" (chap. VIII). Comme on l'a vu, Coray paraît condamner plus universellement l'esclavage. Il cite, à propos de l'influence des lois, une anecdote à cet égard très révélatrice, dans son commentaire au chap. 23 actuel :

Et que ce sont eux seuls qui recueillent l'honneur et le fruit de leurs victoires. Une anecdote très curieuse [...] trouve ici naturellement sa place. Un nègre favori qui avait suivi le colonel Langhedon (qui allait joindre le général Gazes à Sarragoga), lui dit : Maître, vous donnez vous bien du mal, mais vous allez combattre pour liberté. Je souffrirais aussi avec patience, si j'avais liberté à défendre. Qu'à cela ne tienne, reprit Langhedon, dès ce moment-ci, je te la donne. Le nègre le suivit, se conduisit avec courage, et ne l'a pas quitté depuis.

41 Cette anecdote illustre le plus fameux passage du traité Airs, Eaux, Lieux d'une manière peut-être un peu inattendue, mais intéressante, à un moment où la question de l'esclavage était particulièrement brûlante.

Pour compléter encore la contextualisation de l'édition Coray, on peut enfin évoquer l'édition du traité que publia en 1804 un certain Dr Delavaud, médecin aux armées et donc grand voyageur. Le premier, il rend hommage au travail de Coray et veut lui aussi réhabiliter Hippocrate et le rôle qu'il fait jouer à la nature contre «la doctrine mécanico-chimique de Boerhaave ». Cet excessif esprit d'analyse a fait que les médecins « dédaignèrent jusqu'à l'oubli les observations simples ou naturelles des phénomènes des airs, des eaux, des lieux, dans leurs rapports naturels et dans leurs actions combinées sur l'économie de l'homme » (p. XII). En tant que médecin des armées, le Dr Delavaud peut, lui, faire le portrait détaillé de différents peuples du monde et de leur 
caractère en relation avec leur climat et leurs usages. Il relève ainsi l'exemple de la Hollande, typique d'une constitution froide et humide ${ }^{39}$ :

J'ai préféré cet exemple, bien que je regarde l'existence des Hollandais comme une maladie continuelle; mais, en jetant les yeux sur le globe, je n'ai vu, dans un certain état de santé, aucun peuple dans une température froide et humide aussi marquante, et où les usages soient encore plus d'accord avec la température pour en aggraver les maux. Le régime des Anglais est mieux raisonné.

On pourra préférer les observations de Coray, bien qu'une phrase de Delavaud puisse aussi, à mon avis, résumer assez bien leurs deux tentatives : «Le mérite d'Hippocrate ne peut exclure celui de Boerhaave. Seulement l'ancien rectifierait beaucoup le moderne $^{40} . »$

\section{NOTES}

1. Dans la collection Budé (Hippocrate, t. II, 2, Airs, Eaux, Lieux, Paris, Les Belles Lettres [cuf], 1996).

2. Hellénisme et Hippocratisme dans l'Europe méditerranéenne: autour de D. Coray, Actes du Colloque des 20-21 mars 1998, R. ANDRÉANI, H. MICHEL, E. PÉLAQUIER (éd.), université de Montpellier-III, 2000.

3. La Bibliothèque interuniversitaire de médecine (вІUм) en a récemment réalisé la numérisation, qui permet sa consultation aisée (http://www.bium.univ-paris5.fr/histmed/medica/cote? 33163x01 et http://www.bium.univ-paris5.fr/histmed/medica/cote?33163x02).

4. É. Littré, đuvres complètes d'Hippocrate, t. 2, Paris, 1840, p. 11.

5. J. JOUANNA [cf. n. 1], p. 163.

6. "Coray et Hippocrate", dans Hippocrate et son héritage. Colloque franco-hellénique d'Histoire de la médecine, Fondation Marcel Mérieux, Lyon, 1985, p.181-196, et "Place et rôle de Coray dans l'édition du traité hippocratique des Airs, Eaux, Lieux", in D. GouREvitch (éd.), Médecins érudits de Coray à Sigerist, Paris, 1995, p. 7-24.

7. Chap. XIX, 2 Jouanna.

8. Les parallèles que propose Coray entre le médecin, Hérodote $(\mathrm{V}, 10)$ et Aristote (Météor. II, 5 , 362b9) peuvent s'expliquer par des emprunts, notamment pour Aristote, qui connaît certains textes de la Collection hippocratique, mais, en tout cas pour ce qui concerne le médecin et Hérodote, les désaccords qu'on observe aussi en d'autres cas montrent qu'il s'agit plutôt, quand ils sont d'accord, de la reprise par les deux auteurs d'un enseignement sur le climat et son influence qui était répandu dans la seconde moitié du ve siècle.

9. J. JOUANNA [cf. n. 1], p. 233, n. 5.

10. P. 296.

11. P. CLXXVI.

12. P. CLXX-CLXXI du «Discours préliminaire ».

13. Dès les premières lignes du « Discours préliminaire ».

14. P. CLXXIII.

15. L'appel à un approfondissement de la connaissance de la géographie de la Grèce et de la Méditerranée par les Grecs, dans une perspective nationale et éclairée, correspond à un intérêt

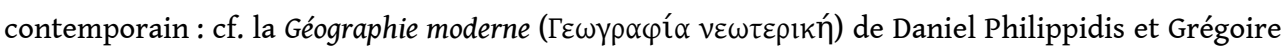
Constandas, en 1791 (je dois cette observation à Mme Danaé Lazaridis, que je remercie vivement). 
16. P. CLXXVII-CLXXX.

17. Extraite de l'Anthologie grecque, IX, 75.

18. Lu à la Société des observateurs de l'homme le 16 Nivôse an XI (6 janvier 1803) par Coray, docteur en médecine et membre de ladite Société.

19. P. 112-113.

20. Cf. c. XVI, 5, p. 229-230 JOUANNA.

21. Cf. c. XXIII-XIV, p. 243-244 JOUANNA.

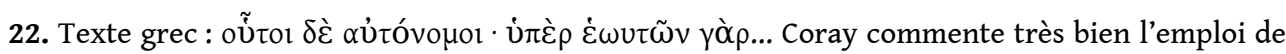
la particule $\gamma \grave{\alpha} \rho$ et propose (p. 376 du Commentaire) de corriger éventuellement oũ̃ol (codd.) en Ǒбol, correction adoptée par les éditeurs suivants et confirmée par la traduction latine.

23. P. 158.

24. Cet article est en effet issu d'une conférence présentée à l'université de Genève.

25. P. 374.

26. P. XXIII-XXIV.

27. Voir notamment J. JOUANNA [cf. n. 1], p. 229 n. 5 et p. 68-69.

28. P. 34-36.

29. Voir la traduction française de la seconde édition, 1815-1820 (t. VI, p. 523 sqq.).

30. Dans son t. 2, « De la Renaissance aux Lumières », Paris, Seuil, 1997.

31. P. LXXXIV.

32. P. 47.

33. P. LXXXV-LXXXVII.

34. Cf. M. D. GRMEK [n. 30], p. 169 Si « le prétendu "retour à Hippocrate" n'est qu'un habile cri de guerre, une illusion savamment entretenue par Sydenham et ses partisans » (en fait disciples de Bacon; et distinguant la maladie des cas individuels, "porteurs de la maladie»), Sydenham « rejoint la tradition épidémiologique de l'hippocratisme classique par sa quatrième règle: "Enfin, on doit remarquer soigneusement les saisons qui favorisent le plus chaque genre de maladie".»

35. MONTESQUIEU, Pensées $n^{\circ} 889$.

36. P. III.

37. P. XXXIV-XXXV.

38. P. XVII.

39. P. CXII.

40. P. 88.

Cette étude est issue d'une conférence prononcée à l'université de Genève le lundi 14 décembre 2009 à l'invitation du professeur Paul Schubert, que je remercie vivement.

\section{RÉSUMÉS}

L'édition du traité hippocratique Des airs, des eaux et des lieux qu'Adiamantos Coray a publiée en 1800 à Paris est un travail philologique novateur. C'est aussi l'œuvre d'un émigré fier de son pays natal, hostile au despotisme asiatique. Docteur en médecine de la Faculté de Montpellier, partisan d'une forme de retour à Hippocrate, Coray doit aussi être situé par rapport à Montesquieu sur la question du déterminisme climatique et de l'esclavage. 
The edition of the Hippocratic treatise of airs, waters and places which Adiamantos Coray published in Paris in 1800 is a groundbreaking philological work. It is also the work of an emigrant proud of his native country and hostile to Asiatic despotism. Doctor of the Montpellier School of Medicine, he advocated a form of return to Hippocrates. Coray must also be assessed in comparison with Montesquieu on the question of climatic determinism and slavery.

\section{INDEX}

Keywords : Hippocrates, hippocratism, Coray Adiamantos, philology, medicine, climate, Ionia, Turkey, despotism, slavery, Montesquieu

Mots-clés : Hippocrate, hippocratisme, Coray Adiamantos, philologie, médecine, climat, Ionie, Turquie, despotisme, esclavage, Montesquieu

\section{AUTEUR}

\section{PAUL DEMONT}

Professeur de langue et littérature grecques

Université de Paris-Sorbonne (Paris-IV)

Paul.Demont@paris-sorbonne.fr 